\title{
RECONSTRUCTION OF 3D ACCIDENT SCENE FROM MULTIROTOR UAV PLATFORM
}

\author{
M.A. Mat Amin ${ }^{1}$, S. Abdullah ${ }^{2}$, S.N. Abdul Mukti ${ }^{3}$, M.H.A. Mohd Zaidi ${ }^{1}$, K.N. Tahar ${ }^{1 *}$ \\ ${ }^{1}$ Centre of Studies for Surveying Science and Geomatics, Faculty of Architecture, Planning and Surveying, Universiti Teknologi \\ MARA, 40450 Shah Alam, Selangor, Malaysia - advancedphotogrammetry@gmail.com; hafizaizuddin21@gmail.com \\ nizamtahar@gmail.com* \\ ${ }^{2}$ Faculty of Architecture, Planning and Surveying, Universiti Teknologi MARA, Seri Iskandar Branch, Perak Campus, 32610 Perak, \\ Malaysia-suzan156@perak.uitm.edu.my \\ ${ }^{3}$ Kuala Lumpur City Hall, Menara DBKL1, Jalan Raja Laut, 50350 Kuala Lumpur - shahrulnizan58@gmail.com
}

\section{Commission II}

KEY WORDS: Accident, Aerial, Point of Interest, UAV, 3D Model, Accuracy

\begin{abstract}
:
Traffic accidents are one of the major causes of fatality in developing countries. The aim of the study is to reconstruct accident scenes by using UAV photogrammetry. The methodology of this study is organised into four main phases which consist of preliminary work, flight planning, 3D model processing and analysis of the results. The 3D model was successfully generated by using Point of Interest (POI) flight planning. The 3D model showed that the results of the process produced good 3D texture where the two vehicles had good shapes and could be seen clearly from an oblique view. In addition, the effect of the tyres on the road could also be seen clearly and had good shape which were generated accurately. The accuracy values obtained from the POI technique and waypoint technique were $0.059 \mathrm{~m}$ and $0.043 \mathrm{~m}$, respectively. Due to the availability of UAVs in the market at reasonable costs, photogrammetry offers the best alternative technique to other methods that have been used to reconstruct the accident scene.
\end{abstract}

\section{INTRODUCTION}

Police used to spend a lot of time preparing documentation by conventional methods (the triangular method, rectangular measurement) by using surveyor's wheel and steel tapeline for the documentation of the accident scene before the use of laser scanners and total stations (Stáňa et al., 2017; Cawood et al., 2017). The photogrammetric reconstruction of the scene starts with image acquisition where images must have particular requisites, not only in terms of object coverage, but also in spatial distribution of camera stations which should follow an ad hoc geometry. The extraction of 3D coordinates requires visibility of the same point scene from at least two points of view by an overlap between consecutive images (Barazzetti et al., 2012; Morales et al., 2015). Accident Reconstruction (AR) is a common term used in the 3D mapping of traffic accident scenes. The first step in AR is characterising the dimensions of the accident accurately. The final output of AR is computer aided drawing, which can be developed into a 3D model or computer animation. The workflow for the 3D reconstruction and energy analysis of the road accident scene is the first thing that needs to be established in this process (Stáňa et al., 2017; Ajayi et al., 2017).

The acquisition of data has to follow two protocols, that are parallel protocol and convergent protocol. Image pre-processing is also needed due to the light conditions at the time of the accident, the presence of shadows, texture, and high specular surfaces along the scene (Jin-Tsong et al., 2015; Fraser, 2008). The purpose is to homogenise the different images captured for the $3 \mathrm{D}$ reconstruction, improving the key point of extraction and matching. The result covers the scale, rotation, and movement between images in an invariant algorithm (Morales et al., 2015). In addition, the advantage of localisation (geodetic total station) is that it is possible to create a $3 \mathrm{D}$ profile of the traffic accident scene including its surrounding to be used for process analysis and to view conditions involved in the traffic accident (Stáňa et al., 2017). Figure 1 shows a 3D Model created in PhotoModeler Scanner software with textures. These photographs were taken around the car, from different angles, with the object being centrally located, having an overlap of about $25 \%$ to $60 \%$. The detail points and lines together with the artificial targets placed on the vehicle body were identified and correlated with the "Reference Mode" software's function. The final 3D model can be visualised with the created surfaces or in a textured mode with the software's high-quality option (Oniga et al., 2016).

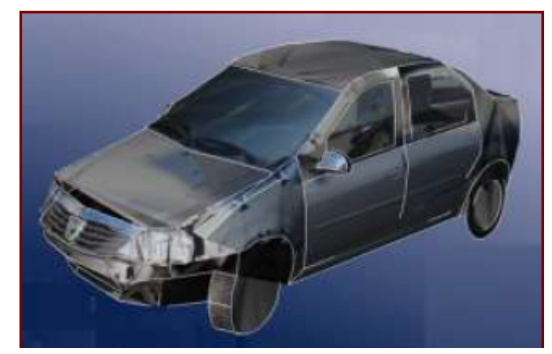

Figure 1. 3D Model created in PhotoModeler Scanner software with textures (Oniga et al., 2016)

The total station has a long history of being used as data by the court for trials, but laser scanning is the recent method used in court documentation (Dustin \& Liscio, 2016). Meanwhile, the advanced method uses geodetic total station and photography which either uses selected points for subsequent rectification or uses an image from UAV for documentation of accident scene. In this case study, the situation of the accident scene makes conventional methods irrelevant as they consume too much time. In addition, the condition of the traffic accident scene and the shape of the intersection impaired makes it hard to use the conventional methods (Alidoost \& Arefi, 2015). The advanced

\footnotetext{
* Corresponding author

K.N Tahar, nizamtahar@gmail.com
} 
method was applied using a geodetic total station to localise the scene area. The advantage of this localisation was that it was possible to create a $3 \mathrm{D}$ profile of the traffic accident scene including the surroundings to be used for process analysis and view the conditions involved in the traffic accident. Chiabrando et al. (2017) said the advantage of UAV images over a rectified image is that it can capture a larger accident scene or provide outlook of the whole scene rather than just limited scene areas, such as those used for viewing collision scenes or in otherwise difficult terrain (fields of grain, bushes, tree). The accident scene also used the geodetic total station to localise, taking 40 minutes and rectified image was applied rather than UAV image because one of the accidents occurred under the railway bridge where UAV imaging was impossible to apply. The advanced method resulted in less time-consuming documentation and subsequent accident scene documentation and also produced the best results in terms of the accuracy of data to be analysed (Stáňa et al., 2017; Osman \& Tahar, 2016; Barazzetti, et al., 2012).

UAVs can be directly fitted with different types of cameras and camcorders (Kamarudin \& Tahar, 2016). When an accident happens, it is possible to get photographic images or video recordings of the traffic accident scene immediately. In addition, documentation can be obtained even after removal of the vehicles from the accident scene in the case where traces of the accident site and the vehicles final positions are appropriately highlighted (Terpstra et al., 2016). Other than that, UAVs can have multiple applications in an investigation scene, such as photography, videotaping, search for evidence, safety assessment, examination of sites which are difficult or near impossible to reach. Before visiting the scene, UAVs can be used to minimise contamination and examine the particular scene (Rychard \& Karpina, 2016). In addition, there were previously only several methods to capture image from an aerial view such as climbing the ladder of a firefighting truck, from a high rise building, or using aircrafts that are costly and require professional handling. UAVs also can be used for forensic purposes where it obtains real-time high-quality images of the scene. UAV also can shorten the period of road closures, thus avoiding traffic congestion, as it quickly assesses the scene. Further, UAVs can save costs in carrying out the crime scene investigation (Tahar, 2015). Lastly, UAV can help to gain access to and retrieve evidence from the accident area where investigators themselves may find it hard (Mendis et al., 2017). Recent advanced methods are expensive for investigators, such as laser scanning and Lidar, compared to UAV, which needs professionals to handle the instruments. Any mistakes made can bring huge costs to the organisation and require time to restart the process. Current methods, such as total station and laser scanning also require road closure for safety. The aim of this study is to reconstruct accident scenes with UAV photogrammetry.

\section{MATERIAL AND METHODS}

The methodology of this study is organized into four main phases. The first phase is about preliminary work which consists of site selection, software selection, and instrument selection. Meanwhile, the second phase is about data acquisition that involved in this study such as flight planning Point of Interest (POI) and Waypoint (Aerial Nadir), and camera calibration. The third phase is about data processing which included image processing, image dense cloud, image meshing, image texturing and 3D model processing at the end of process data. Lastly, the fourth phase is about result and analysis which accuracy assessment and a frequency table are conducted.

\subsection{Preliminary Work}

The more data and information that characterizing the accident area allows a best 3D Representation of that area. It is important to determine the flying altitude, point of take-off, and point landing safely for UAVs. In this research, geographical features were an important factor to be considered to ensure there were no disturbances during the flight mission. The terrain information of the study area was needed to ensure the safety of the UAV flight during the data acquisition process. After going through several aspects, the study area was chosen at Canseleri carpark, Universiti Teknologi MARA Shah Alam as shown in Figure 2.

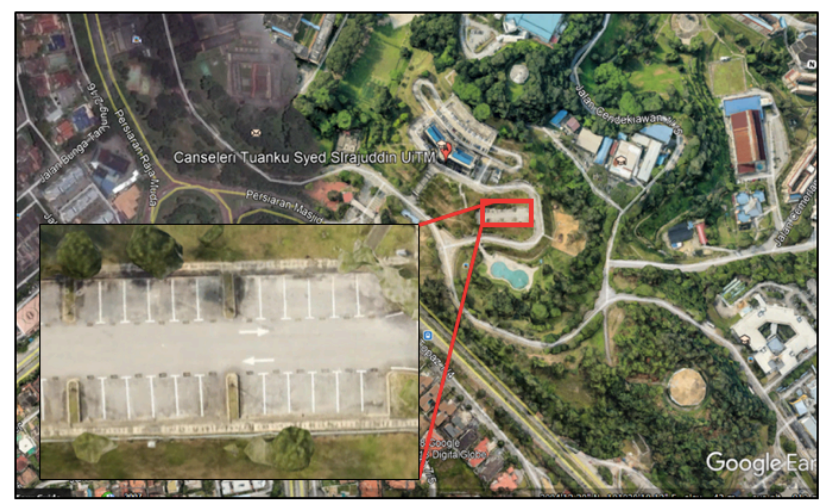

Figure 2. Car Park at Study Area

\subsection{Data Acquisition}

There are many flight planning apps can be used for UAV's flight mission. However, this study used DJI GO 4 and Altizure apps to design the flight plan and for point of interest and waypoint techniques. Flight planning for the DJI Phantom 4 uses the DJI GO 4 app which captured images of the accident scene. The DJI GO app is limited for use by the DJI Phantom 3 Pro. The setting for the flight altitude was 15 meters and the radius surrounding the accident scene was 15 meters. The images were captured manually by the operator every two seconds while the drone flew surrounding the accident scene with horizontal speed of $1.0 \mathrm{~m} / \mathrm{s}$. The overlapping images was about $90 \%$. Figure 3 a shows the 38 images of POI technique. Flight planning was the first step to planning and simulate a mapping mission. In the autonomous capturing mode, the Altizure app controlled the DJI drones with the help of DJI SDK to capture vertical photographs. The flight planning was important to avoid any misconduct during the work process. There were several steps to follow before establishing the flight planning which were to make sure the latest firmware was installed on the aircraft and that the phone was connected to the Internet when launching the Altizure app for the first time.

Before launching the app, the drone and remote controller were turned on. The Flight Mode Switch on the controller (labelled PA-F) was set to F-Mode. A rectangular region to cover and the position, size, and direction were chosen. The safety of flight paths was also checked to ensure there was no obstacle along the path. Figure $3 \mathrm{~b}$ shows the flight planning of the study area with area $30 \mathrm{~m} \times 25 \mathrm{~m}$. The waypoints technique used multiple overlapping photos with $80 \%$ to $90 \%$ overlap and were taken by an autonomous programmed flight path. There were 29 images were captured by waypoints technique in this study. Generally, information on weather conditions is necessary to avoid cloudy or strong wind. In waypoint flight planning, a vertical flight based on nadir images was taken with grid flight plans achieving high overlaps and eliminated systematic errors. With a flying height 
of approximately 15 meters above the ground, therefore, the calculated ground sampling distance about $6 \mathrm{~mm}$. The size of image footprint on the ground is about $25.6 \mathrm{~m} \times 19.2 \mathrm{~m}$.

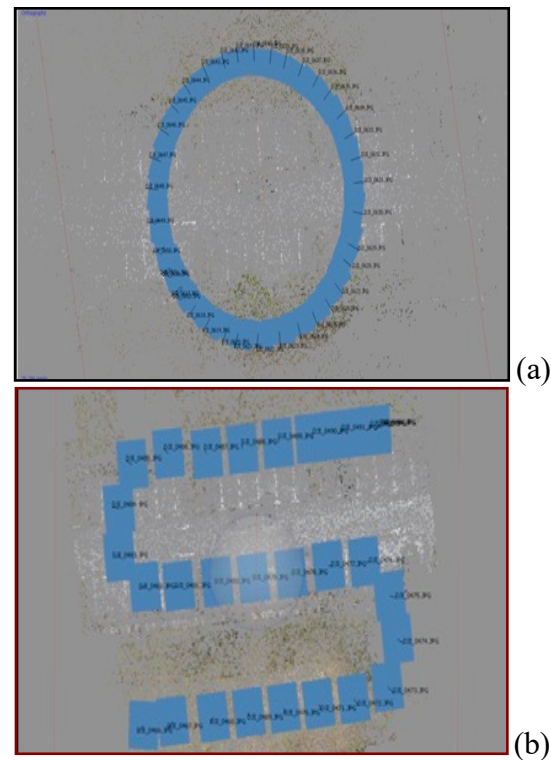

Figure 3. Flight planning; (a) Waypoint technique, (b) POI technique

\subsection{Data Processing}

This study used commercial software known as Agisoft Metashape to process all acquired images from the UAV platform. During software processing, there were several steps that needed to be followed to obtain a 3D model, which were to align the photos, build a dense cloud, build mesh, and build texture (Figure 4). Before starting any operation, it is necessary to point out what photos will be used as a source for $3 \mathrm{D}$ reconstruction. The photos were then loaded into the software after making a detailed choice of images from the folder. Metashape supports processing of images saved as multichannel (single page) TIFF files. The main processing stages for images were performed based on the master channel, which were selected by the user. During the orthophoto export, all spectral bands were processed together to form a orthophoto with the same bands as the source images.

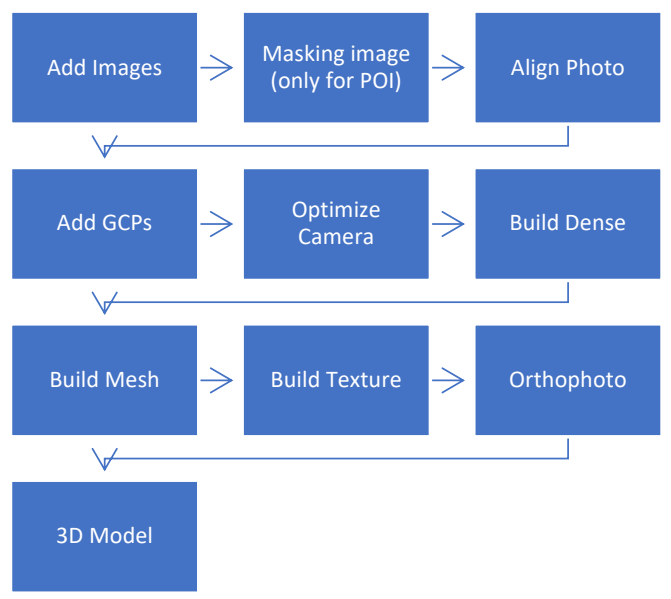

Figure 4. General methodology using Agisoft Metashape additional master channel selection step performed after adding images to the project. For the best results it is recommended to select the spectral band which is sharp and with as much detail as possible. For POI data processing, all 38 images were selected to process the data from the accident scene. Before aligning the photo, the images needed to mask to concentrate on the object of interest. The masking was done by selecting the intelligent scissors icon, selecting an image and choosing the area to mask. The mask area was surrounded by red dots, and the line was automatically masked with each other with the escape key as shown on the Figure 5a. The invert selection icon ensured the image selected was the image of accident scene and saved the masking setting on the images (Figure 5b). Once the photos were loaded into Metashape, it needed to be aligned. At this stage Metashape found the camera position and orientation for each photo and built a sparse point cloud model. The accuracy of the aligned photos was selected as high and the pair pre-selection was generic.
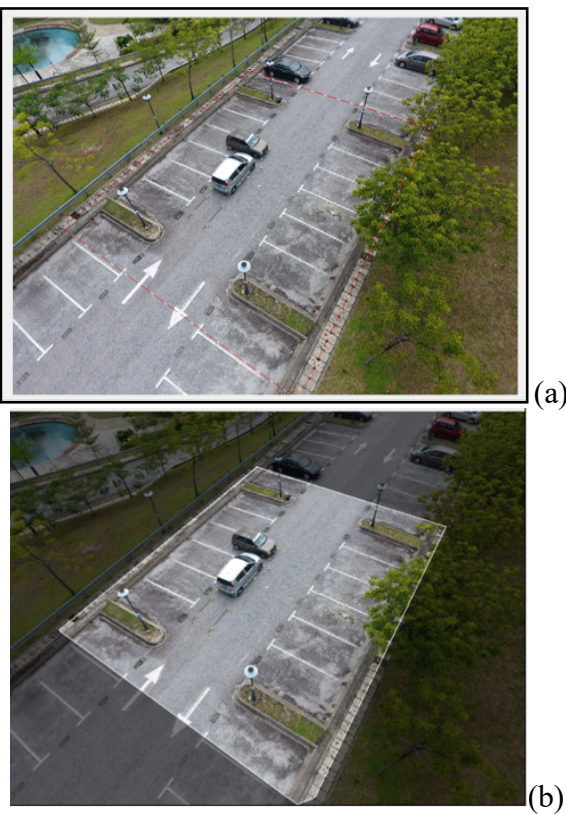

Figure 5. Mask the accident area; (a) masking line, (b) invert selection after masking

When the alignment step is completed, the point cloud and estimated camera positions can be exported for processing with another software if needed. After aligning the photo, markers were created on the image as Ground Control Point (GCP); GCP 1, GCP 2, GCP 3, and GCP 4. Each GBP needed at least 3 markers on the images such as GCP 1 which had 3 markers on different images. The place of markers are shown on Figure 6. After markers were completed, the coordinates from the text file were imported to input in the processing. The coordinate system was changed to Kertau RSO Malaya (m) using the icon import tool to open the coordinate input settings.

The overall procedure for imagery processing does not differ from the usual procedure for normal photos, except for the 


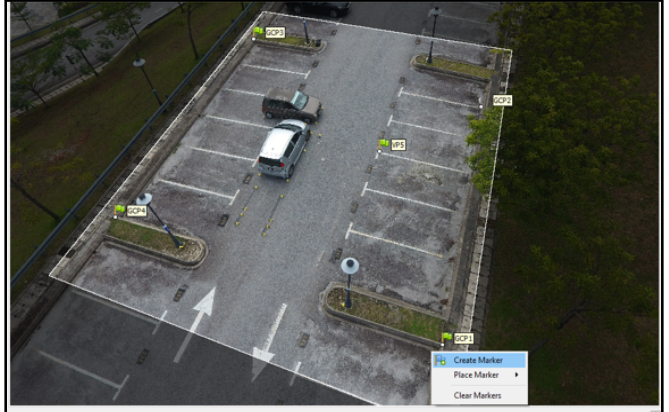

Figure 6. Place of markers

Optimising the camera is important in photogrammetry processing to reduce the error during data processing. Optimising the camera is done by unchecking all the images selected for the processing. The settings chosen for the camera alignment were fit f, fit cx, cy, fit k1, fit k2, fit k3, fit b1, fit b2, fit p1, and fit $\mathrm{p} 2$. A dense cloud was built, and the redundancy of disparity was used for blunder detection and to improve the accuracy of the final depth maps due to existing high overlap between images. Metashape allowed a dense point cloud to be generated and visualized. The program calculates depth information for each camera to be combined into a single dense point cloud. Metashape tends to produce extra dense point clouds, which are of almost the same density. A dense point cloud can be edited and classified within the Metashape environment or exported to an external tool for further analysis. This study used a high quality build dense cloud with mild depth filtering. The next step was the 3D model which comprised mesh generation and refinement, and texture mapping.

Mesh refinement was divided into two steps, the first being to have a surface covering the whole area to model based on Delaunay 2.5D triangulation. The setting for the build mesh was arbitrary, the data was chosen from the dense cloud and the face count was high at 327,661 . The results of this step was that some small mesh merged into the bigger parts based on their area size. Then, the rough mesh was refined based on the deviation error between the mesh and the estimated best shape to generate lightweighted and accurate polygonal models. The deviation error was calculated with local curvature. The final step in the 3D model was texture mapping or multi-texturing which was assigned to each triangle in the $3 \mathrm{D}$ model, so the misalignment between textures from different sources could be very small. The texture mapping mode determines how the object texture will be packed in the texture atlas. Proper texture mapping mode selection helps to obtain optimal texture packing and, consequently, better visual quality of the final model. The generic setting for build texture was chosen for the mapping mode. Figure 7a shows the result of the completed build texture which was generated as a 3D model. The 3D model was successfully generated with POI flight planning which showed the model was smooth.

For waypoint processing, all 29 images were selected to process data of the accident scene. Once photos were loaded into Metashape, they needed to be aligned. At this stage Metashape finds the camera position and orientation for each photo and builds a sparse point cloud model. The accuracy of the aligned photos was selected as high. When the alignment step was completed, the point cloud and estimated camera positions can be exported for processing with another software if needed. All the 29 images were aligned and distributed by waypoints flight planning. After the alignment was completed, the coordinates from the text file was imported to input the coordinate system into Kertau RSO Malaya (m). The coordinate system automatically matched with the markers that were established earlier. The setting that been choosen for the optimize camera alignment same as POI processing. This study used the setting of build dense cloud which the quality chooses high in the process and the depth filtering is aggressive.

The setting for the build mesh on the surface type is height field which different from the POI setting earlier. The source data choose the dense cloud and the face count choose high. The setting for the build texture on the mapping mode is choose orthophoto which different setting from the POI. Metashape estimates image quality as a relative sharpness of the photo with respect to other images in the data set. The value of the parameter is calculated based on the sharpness level of the most focused part of the picture. Figure $7 \mathrm{~b}$ shows the completed build texture.

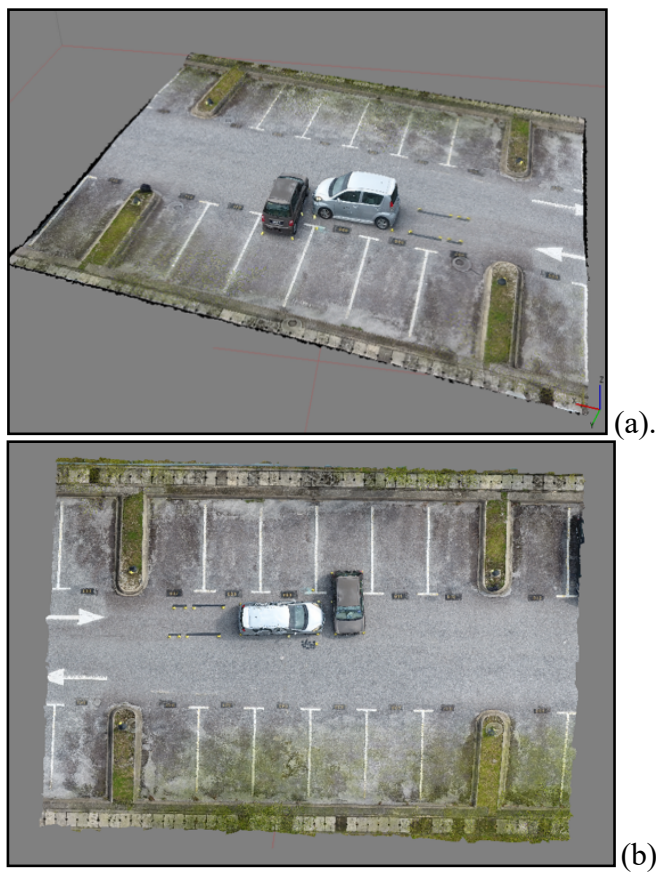

Figure 7. Build Texture; (a) POI, (b) Waypoint

\section{RESULTS AND ANALYSIS}

The GCPs coordinates for this study are as illustrated in Table 1 . The GPS observations were conducted in the accident area. There are four GCPs established in this study. The GPS observation coordinate system used is Malaysian Real-Time Kinematic GNSS Network (MyRTKnet) and geodetic datum is Geocentric Datum for Malaysia 2000 (GDM2000) and the map projection system used in the Agisoft Photosan software Malayan Rectified Skew Orthomorphic (Peninsular Malaysia).

Table 1. GCP at accident scene area

\begin{tabular}{cccc}
\hline Control & Northing & Easting & Height \\
\hline GCP 1 & 339829.570 & 389799.433 & 35.6430 \\
GCP 2 & 339831.137 & 389812.536 & 35.4390 \\
GCP 3 & 339846.681 & 389813.534 & 35.6336 \\
GCP 4 & 339845.317 & 389798.932 & 35.5983 \\
\hline
\end{tabular}

The final product for POI technique that produce by the software as shown in Figure 8a is 3D texture mapping of traffic accident scene. The $3 \mathrm{D}$ model shows that the processing results are able to produce $3 \mathrm{D}$ with good texture where the two vehicles have good shape and the shape of vehicles can be seen clearly from the oblique view. In addition, the tyre effects on the road can also be seen clearly and have good shape and generated accurately. The 
black debris on the road can been seen near the silver vehicle while the white debris near the brown vehicle could not been seen clearly from an oblique view. The four road lamp have been used as land mark for this accident scene where results showed that the only the base and head of the road lamp could be seen on the 3D model.

The final product for waypoint technique produced by the software as shown in Figure $8 \mathrm{~b}$ was texture mapping of traffic accident scene. The 3D model showed that the result of the processing was able to produce good texture where the two vehicles have a good shape and the shape of vehicles can be seen clearly from the nadir view. In addition, the effect of the tyres on the road could also be seen clearly and had a good shape and generated accurately. The black debris on the road can been seen which near the black vehicle meanwhile the white debris which near the brown vehicle cannot been seen from a nadir view. The road lamp has been used as land mark for this accident scene which the result shows that the road lamp cannot be generated properly and only the base of the road lamp can be seen on the 3D model.

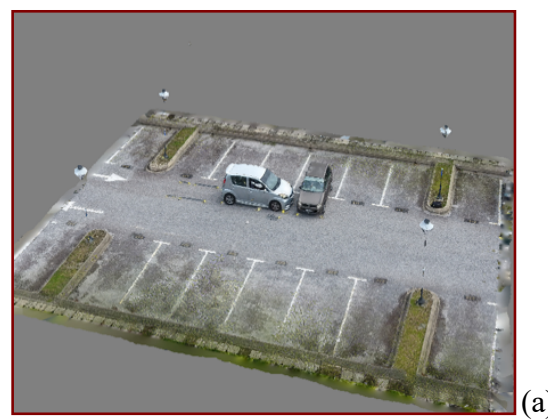

(a)

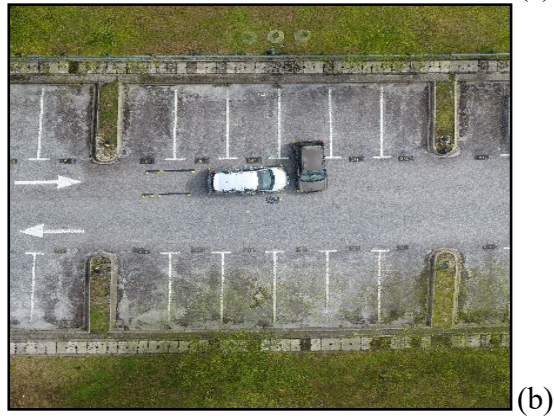

(b)

Figure 8. 3D Model of accident scene; (a) POI, (b) Waypoints

The accuracy assessment analysis used 33 points to measure a few sample of distances. The same distances also are measured using tools in Agisoft Metashape. These two types of measurements were compared with the same distances calculated directly from the points. Figure 9 shows the images of accident scene taken by a police investigator with a digital camera. Every detail at the accident scene needs to be captured for investigation and to model the accident by forensics at the laboratory.

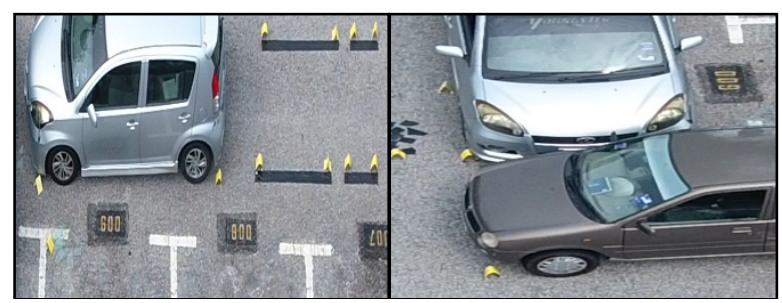

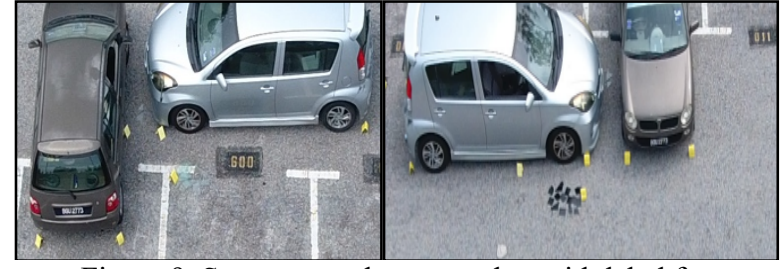

Figure 9. Some examples on marker with label for investigations

Then, the measurements are taken at the accident scene. Measurements are taken by tape, or more recently, lasers from smartphones are used. Road lamps were used as land marks for this study with the series numbers used as a reference. In addition, if the accident scene did not have a road lamp, buildings were used as land marks. If the accident happened on a highway, the KM (kilometre) label at the centre of highway is used as a land mark. There have been several measurements taken by police on the accident scene, such as tyre marks, the distance between two vehicles, debris distance with vehicle and the distance of vehicles from the land mark (road lamp). Only one land mark was used in the investigation of police. Figure 10 shows the measurements taken by police investigators at an accident area. There were 13 measurements in total indicated by red lines.

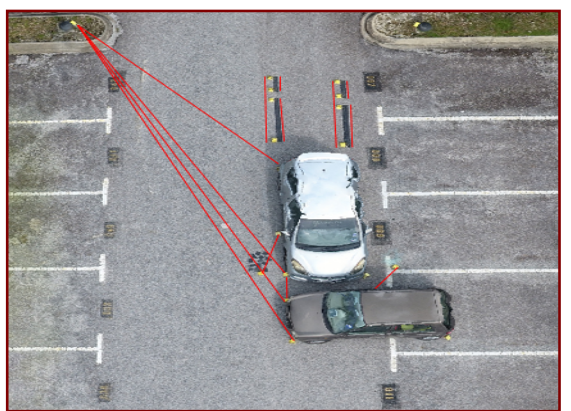

Figure 10. Measurement taken by Police Investigator

The speed of the vehicles can be known by the length of the tyre on the road. The longer the tyre effect the higher the vehicle speed, as the vehicle takes time to stop and causes marks on the road. This study analyses the behaviour of the results with a class frequency table. A class frequency table was done with data from UAV measurements with 33 samples obtained from POI and waypoint techniques. For POI results, Figure 11a shows that the data is skewed to the left where the highest values of class relative frequency was in the range of 0.583 to 3.803 and the lowest values of class relative frequency was in the range of 10.243 to 11.850. For waypoint results, Figure $11 \mathrm{~b}$ shows the data was skewed to the left where the highest values of class relative frequency was in the range of 0.526 to 3.774 and the lowest values of class relative frequency was in the range of 10.270 to 11.894 . 


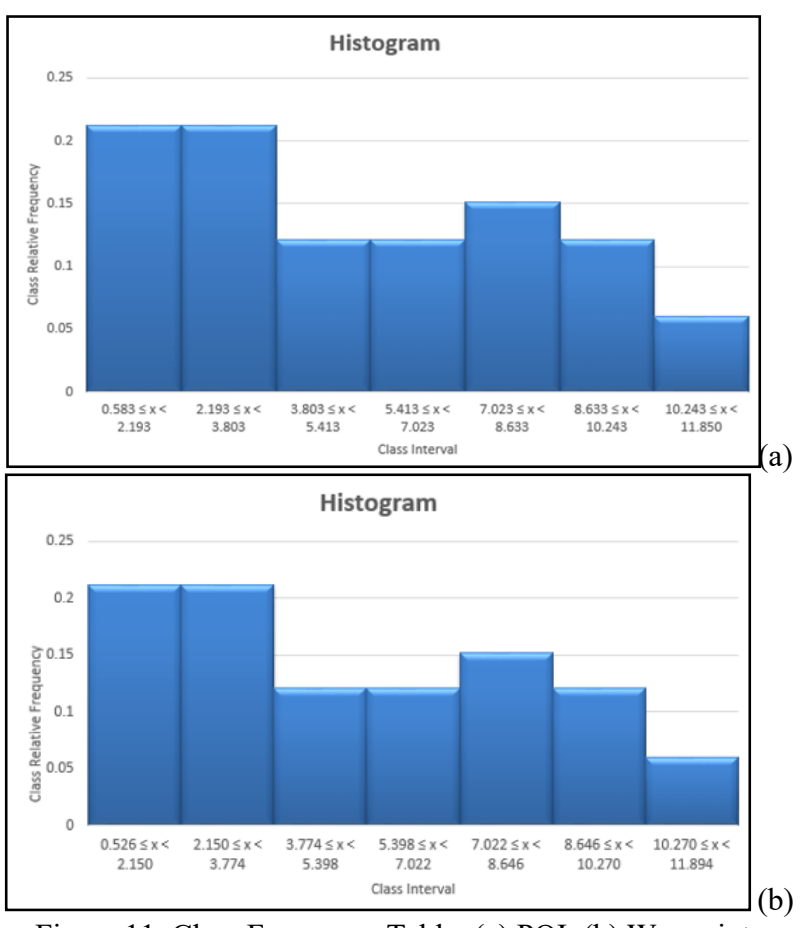

Figure 11. Class Frequency Table; (a) POI, (b) Waypoints

The accuracy assessment of this study was based on the comparison between actual measurements using total station and the UAV products measurements from the Agisoft Metashape software. Table 2 and Table 3 show the comparison of actual and measured values on POI and waypoints, respectively, based on 33 samples. The standard deviation has been calculated to determine the range of acceptable data for this study. The data outside the standard deviation range were known as outlier data which cannot be used for the Root Mean Square Error (RMSE) calculation. The RMSE can determine the accuracy of UAV products in this study. Therefore, the range of errors for POI technique are $-0.281 \mathrm{~m}$ to $0.040 \mathrm{~m}$. The standard deviation for POI technique was $\pm 0.066 \mathrm{~m}$.

\begin{tabular}{cccc}
\multicolumn{5}{c}{ Table 2. Errors for POI technique } \\
\hline $\begin{array}{c}\text { Sample } \\
\text { Distance }\end{array}$ & $\begin{array}{c}\text { Site } \\
\text { measurement } \\
(\mathrm{m})\end{array}$ & $\begin{array}{c}\text { UAV } \\
\text { measurement } \\
(\mathrm{m})\end{array}$ & $\begin{array}{c}\text { Error } \\
(\mathrm{m})\end{array}$ \\
\hline $\mathbf{1}$ & 7.412 & 7.360 & -0.052 \\
$\mathbf{2}$ & 8.361 & 8.336 & -0.025 \\
$\mathbf{3}$ & 9.860 & 9.757 & -0.103 \\
$\mathbf{4}$ & 8.979 & 8.874 & -0.105 \\
5 & $\mathbf{1 1 . 8 1}$ & $\mathbf{1 1 . 8 5 0}$ & $\mathbf{0 . 0 4 0}$ \\
$\mathbf{6}$ & 6.260 & 6.215 & -0.045 \\
$\mathbf{7}$ & 5.493 & 5.439 & -0.054 \\
$\mathbf{8}$ & 8.536 & 8.436 & -0.100 \\
$\mathbf{9}$ & 8.122 & 8.085 & -0.037 \\
$\mathbf{1 0}$ & 4.912 & 4.833 & -0.079 \\
$\mathbf{1 1}$ & 5.652 & 5.597 & -0.055 \\
$\mathbf{1 2}$ & 4.326 & 4.274 & -0.052 \\
$\mathbf{1 3}$ & 3.050 & 3.034 & -0.016 \\
$\mathbf{1 4}$ & 2.848 & 2.835 & -0.013 \\
$\mathbf{1 5}$ & 2.370 & 2.360 & -0.010 \\
$\mathbf{1 6}$ & 4.868 & 4.826 & -0.042 \\
$\mathbf{1 7}$ & 3.872 & 3.823 & -0.049 \\
$\mathbf{1 8}$ & 3.762 & 3.692 & -0.070 \\
$\mathbf{1 9}$ & 3.478 & 3.417 & -0.061 \\
20 & $\mathbf{6 . 8 5 6}$ & $\mathbf{6 . 6 3 1}$ & $\mathbf{- 0 . 2 2 5}$ \\
21 & $\mathbf{7 . 9 6 6}$ & $\mathbf{7 . 7 8 3}$ & $\mathbf{- 0 . 1 8 3}$ \\
$\mathbf{2 2}$ & $\mathbf{9 . 6 2 0}$ & $\mathbf{9 . 4 5 3}$ & $\mathbf{- 0 . 1 6 7}$ \\
\hline & & &
\end{tabular}

\begin{tabular}{cccc}
\hline 23 & $\mathbf{1 0 . 2 9 6}$ & $\mathbf{1 0 . 0 1 5}$ & $\mathbf{- 0 . 2 8 1}$ \\
24 & $\mathbf{1 1 . 4 1 0}$ & $\mathbf{1 1 . 2 4 0}$ & $\mathbf{- 0 . 1 7 0}$ \\
$\mathbf{2 5}$ & 0.678 & 0.636 & -0.042 \\
$\mathbf{2 6}$ & 1.310 & 1.197 & -0.113 \\
$\mathbf{2 7}$ & 0.560 & 0.538 & -0.022 \\
$\mathbf{2 8}$ & 2.250 & 2.203 & -0.047 \\
$\mathbf{2 9}$ & 1.492 & 1.451 & -0.041 \\
$\mathbf{3 0}$ & 2.276 & 2.220 & -0.056 \\
$\mathbf{3 1}$ & 0.630 & 0.610 & -0.020 \\
$\mathbf{3 2}$ & 1.432 & 1.381 & -0.051 \\
$\mathbf{3 3}$ & 1.066 & 1.006 & -0.060 \\
\hline
\end{tabular}

There are six outliers (bold and highlighted) in the POI results while 82 percent of the data are acceptable for RMSE calculation. The RMSE for the POI technique was $0.059 \mathrm{~m}$ based on 27 acceptable data. The range of error for the waypoint technique were between $-0.159 \mathrm{~m}$ to $0.084 \mathrm{~m}$. The standard deviation for the waypoint technique was $\pm 0.053 \mathrm{~m}$. There were nine outliers (bold and highlighted) in the waypoint results while about 73 percent of the data was acceptable for RMSE calculation. The RMSE for the waypoint technique was $0.043 \mathrm{~m}$ based on 24 acceptable data.

Table 3. Errors for waypoint technique

\begin{tabular}{|c|c|c|c|}
\hline $\begin{array}{c}\text { Sample } \\
\text { Distance }\end{array}$ & $\begin{array}{c}\text { Site } \\
\text { measurement } \\
(\mathrm{m})\end{array}$ & $\begin{array}{c}\text { UAV } \\
\text { measurement } \\
(\mathrm{m})\end{array}$ & $\begin{array}{l}\text { Error } \\
(\mathrm{m})\end{array}$ \\
\hline 1 & 7.412 & 7.363 & -0.049 \\
\hline 2 & 8.361 & 8.356 & -0.005 \\
\hline 3 & 9.860 & 9.845 & -0.015 \\
\hline 4 & 8.979 & 8.882 & -0.097 \\
\hline 5 & 11.810 & 11.894 & 0.084 \\
\hline 6 & 6.260 & 6.280 & 0.020 \\
\hline 7 & 5.471 & 5.545 & 0.074 \\
\hline 8 & 8.536 & 8.594 & 0.058 \\
\hline 9 & 8.122 & 8.144 & 0.022 \\
\hline 10 & 4.912 & 4.879 & -0.033 \\
\hline 11 & 5.652 & 5.582 & -0.070 \\
\hline 12 & 4.326 & 4.308 & -0.018 \\
\hline 13 & 3.050 & 3.035 & -0.015 \\
\hline 14 & 2.848 & 2.815 & -0.033 \\
\hline 15 & 2.370 & 2.345 & -0.025 \\
\hline 16 & 4.868 & 4.836 & -0.032 \\
\hline 17 & 3.872 & 3.812 & -0.060 \\
\hline 18 & 3.762 & 3.668 & -0.094 \\
\hline 19 & 3.478 & 3.405 & -0.073 \\
\hline 20 & 6.856 & 6.746 & -0.110 \\
\hline 21 & 7.966 & 7.934 & -0.032 \\
\hline 22 & 9.620 & 9.562 & -0.058 \\
\hline 23 & 10.296 & 10.137 & -0.159 \\
\hline 24 & 11.410 & 11.374 & -0.036 \\
\hline 25 & 0.678 & 0.639 & -0.039 \\
\hline 26 & 1.310 & 1.151 & -0.159 \\
\hline 27 & 0.560 & 0.526 & -0.034 \\
\hline 28 & 2.250 & 2.228 & -0.022 \\
\hline 29 & 1.492 & 1.464 & -0.028 \\
\hline 30 & 2.276 & 2.251 & -0.025 \\
\hline 31 & 0.630 & 0.593 & -0.037 \\
\hline 32 & 1.432 & 1.402 & -0.030 \\
\hline 33 & 1.066 & 1.017 & -0.049 \\
\hline
\end{tabular}

Figure 12 shows the simulation of the accident scene by used virtual crash demo software. The simulation showed the prediction of vehicle collisions that were reconstructed from the data obtained on the scene. 


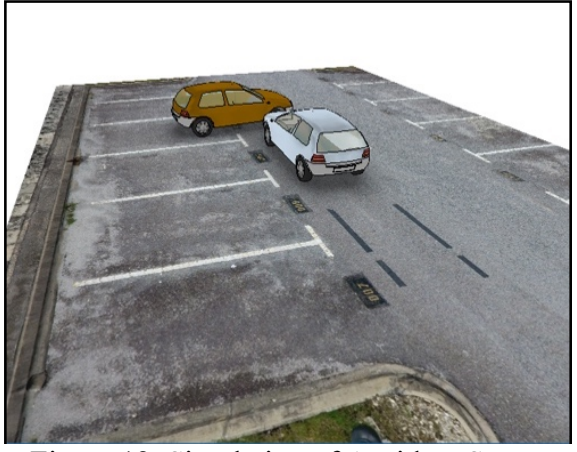

Figure 12. Simulation of Accident Scene

Figure 13 shows a drawing plan of a 3D mapping accident scene where the best accuracy was by waypoints data and the best view of the model was by POI data. The 3D model of the accident scene was able to be produce successfully by UAV flight planning based on POI and waypoint techniques to capture images of the accident scene. The POI technique can provide very fine $3 \mathrm{D}$ models. The waypoint technique is good to visualize the accident scene in $2 \mathrm{D}$ orthophoto which can help police record details at the accident scene for future reference.

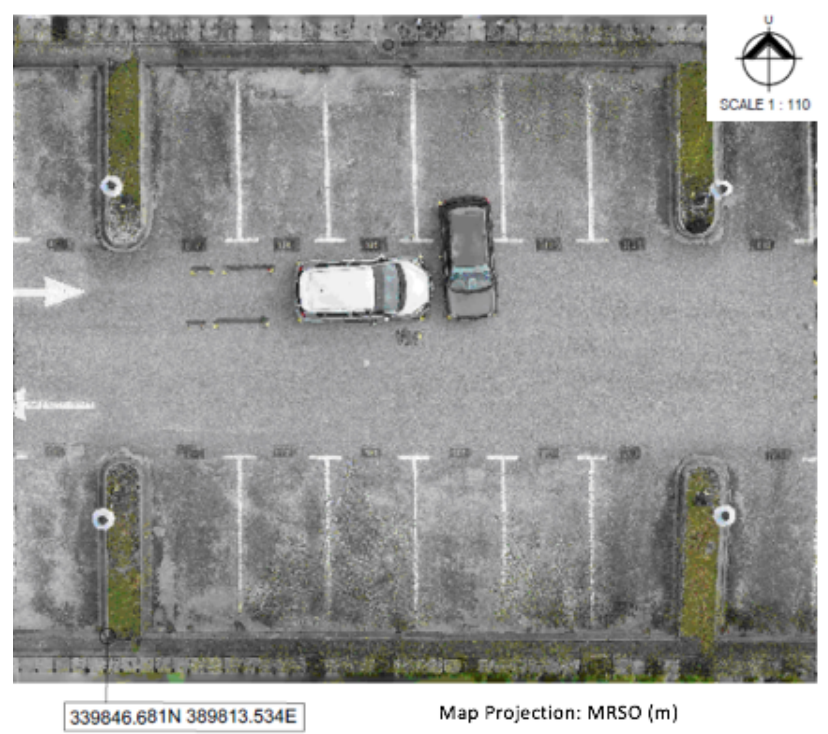

Figure 13. Drawing Plan of 3D Mapping Accident

\section{CONCLUSIONS AND RECOMMENDATIONS}

The 3D accident scene model that was obtained from this study can be used for real accidents. The results demonstrated that the use of UAV photogrammetry can provide accuracy in centimetres either for accuracy of the 3D model or orthophoto for visualisation. The accuracy values obtained from the POI technique and waypoint technique were $0.059 \mathrm{~m}$ and $0.043 \mathrm{~m}$, respectively. Therefore, the waypoint technique resulted in more accurate results than the POI technique. Due to the availability of UAVs in the market at reasonable costs, photogrammetry offers the best alternative technique to any other method that been used to reconstruct the accident scene. Based on this study, the 3D model of accident scene by UAV photogrammetry can speed up the police documentation with reliable accuracy.

\section{ACKNOWLEDGEMENTS}

Faculty of Architecture, Planning, and Surveying Universiti Teknologi MARA (UiTM), Research Management Institute (RMi) and Ministry of Higher Education (MOHE) are greatly acknowledged for providing the fund BESTARI 600IRMI/MyRA 5/3/BESTARI (001/2017) to enable this research to be carried out. The authors would also like to thank the people who were directly or indirectly involved in this research.

\section{REFERENCES}

Ajayi, O. G., Salubi, A. A., Angbas, A. F., and Odigure, M. G. 2017. "Generation of accurate digital elevation models from UAV acquired low percentage overlapping images". International Journal of Remote Sensing, 22-31.

Alidoost, F., and Arefi, H. 2015. "An Image-Based Technique for 3D Building Reconstruction using Multi-View UAV images". International Conference on Sensors \& Models in Remote Sensing \& Photogrammetry, 43-46.

Barazzetti, L., Sala, R., Scaioni, M., Cattaneo, C., Gibelli, D., Giussani, A., Poppa, P., Roncoroni, F., and Vandone, A. 2012. " $3 \mathrm{D}$ scanning and imaging for quick documentation of crime and accident scenes". Proc. of SPIE, 1-14.

Cawood, A. J., Bond, C. E., Howell, J. A., and Butler, R. W. 2017. "LiDAR, UAV or compass-clinometer? Accuracy, coverage and the effects on structural models". Journal of Structural Geology, 67-82.

Chiabrando, F., Lingua, A., Maschio, P., and Teppati Losèa, L. 2017. "The Influence of Flight Planning and Camera Orientation in UAVs Photogrammetry". The International Archives of the Photogrammetry, Remote Sensing and Spatial Information Sciences, 163-170.

Dustin, D., and Liscio, E. 2016. "Accuracy and Repeatability of the Laser Scanner and Total Station for Crime and Accident Scene Documentation". J Assoc Crime Scene Reconstr., 57-67.

Fraser, C., Cronk, S., and Hanley, H. 2008. "Close-Range Photogrammetry in Traffic Incident Management". The International Archives of the Photogrammetry, Remote Sensing and Spatial Information Sciences, 125-128.

Jin-Tsong , H., Ting-Chen , C., and Chien-Hong , C. 2015. "Accuracy Assessment of 3D Building Construction by Multiview Images and the Integrated Application with Augmented Reality". New Taipei City: Ministry of Science and Technology of Taiwan.

Kamarudin, S. S., and Tahar, K. N. 2016. "Assessment on UAV Onboard Positioning in Ground Control Point Establishment". 2016 IEEE 12th International Colloquium on Signal Processing \& its Applications, 210-215.

Mendis , N., Dharmarathne, T., and Wanasinghe, N. 2017. "Use of Unmanned Aerial Vehicles in Crime Scene InvestigationsNovel Concept of Crime Scene Investigations". Forensic Research \& Criminology International Journal, 1-2.

Morales, A., Aguilera, D. G., Gutiérrez, M. A., and López, A. I. 2015. "Energy Analysis of Road Accidents Based on CloseRange Photogrammetry". Remote Sensing, 15161-15178.

Oniga, V. E., Loghin, A.-M. M., and Păun, D. C. 2016. "Crashed vehicle profile creation based on digital Close-range photogrammetry". World Journal of Engineering Research and Technology, 2(4), 16-29. 
Osman, M. R., and Tahar, K. N. 2016. "3D accident reconstruction using low-cost imaging technique". Advances in Engineering Software, 231-237.

Rychard, M. J., and Karpina, M. 2016. "Quality Analysis on 3D Building Models Reconstructed from UAV Imagery". XXIII ISPRS Congress, 1121-1126.

Stáňa, I., Tokař, S., Bucsuházy, K., and Bilík, M. 2017. "Comparison of Utilization of Conventional and Advanced Methods". 10th International Scientific Conference Transbaltica 2017: Transportation Science and Technology, 471 - 476.

Tahar, K. N. 2015. "Efficiency and cost comparison of UAV/Field survey". Proceeding of the 2015 International Conference on Space Science am Communication (IconSpace), 428-433).

Terpstra, T., Voitel, T., and Hashemian, A. 2016. "A Survey of Multi-View Photogrammetry Software for Documenting Vehicle Crush". SAE International, 1-31. 